\title{
Synthesis and characterization of 1,2,4-triazole containing hydrazide-hydrazones derived from (S)-Naproxen as anticancer agents
}

\author{
Muhammed İhsan HAN 1, Hatice BEKÇİ ${ }^{2}$ Ahmet CUMAOĞLU 2, Ş. Güniz KÜÇÜKGÜZEL ${ }^{1}$ * \\ 1 Marmara University, Faculty of Pharmacy, Department of Pharmaceutical Chemistry, Haydarpaşa 34668 İstanbul, \\ Turkey. \\ 2 Erciyes University, Faculty of Pharmacy, Department of Pharmaceutical Biochemistry, Talas 38280 Kayseri, Turkey \\ * Correspondence: gkucukguzel@marmara.edu.tr (S..G.K.) ; Tel. +90-216-414 29 62; ORCID No: 0000-0001-9405-8905
}

Received: 4 April 2018 / Revised: 22 May 2018 / Accepted: 22 May 2018

\begin{abstract}
A novel series of new naproxen derivatives (S)-ethyl\{[4-(4-fluorophenyl)-5-[(1-(6-methoxynaphtalen-2yl)ethyl)]-4H-1,2,4-triazole-3-yl]sulphanyl\}acetate (5), (S)-2-(\{5-[1-(6-methoxynaphtalen-1-yl)ethyl]-4-fluorophenyl-4H1,2,4-triazole-3-yl\}sulphanyl)acetohydrazide (6), 2-\{[5-[1-(6-methoxynaphtalen-2-yl)ethyl]-4-(4-fluorophenyl)-4H-1,2,4triazole-3-yl] sulphanyl\}- $N^{\prime}$-[(substituted)methylidene]acetohydrazides (7a-m) were synthesized, in this study. The structures of compounds 5, 6 and 7a-m were defined by spectral ( ${ }^{1} \mathrm{H}-\mathrm{NMR},{ }^{13} \mathrm{C}-\mathrm{NMR}$, HR-MS and FT-IR) methods and their purity was proven by elemental analysis, thin layer chromatography and high pressure liquid chromatography. These compounds were evaluated for in vitro anticancer activity by using MTS method against PC-3 and DU-143 (androgen-independent human prostate cancer cell lines) and LNCaP (androgen-sensitive human prostate adenocarcinoma) prostate cancer cell lines. Cisplatin was used as the positive sensitivity reference standard. Compounds (7a-m) exhibited anticancer activity with $\mathrm{IC}_{50}$ values of $87.2-400 \mu \mathrm{M}$ against prostate cancer cell lines.
\end{abstract}

KEYWORDS: Anticancer activity; MTS method; (S)-Naproxen; hydrazide-hydrazones.

\section{INTRODUCTION}

Cancer, defined as uncontrolled proliferation and spread of abnormal cells, is one of the most important health problems of our time. It is also a public health problem because of its frequent appearance and high mortality. The rapid rise of cancer cases in the world and in our country and the fact that they are among the first causes of deaths suggests that further studies on the development of new drugs and treatment methods should be conducted in this regard. According to the latest data of association in Turkey fight cancer, is the second highest incidence of cancer among men with prostate cancer was $37.6 \%$.

Non-steroidal anti-inflammatory drugs (NSAIDs) are the most used up medicinal drugs generally used as anti-inflammatory, antipyretic and analgesic drugs and their side-effects were worked involving gastrointestinal and cardiovascular risks [1]. (+)-(S)-2-(6-Methoxynaphthalen-2-yl)propanoic acid (Naproxen) is a non-steroidal anti-inflammatory drug which contains carboxylic acid funcionality with antipyretic and analgesic properties. It is thought to study by reducing the action of cyclooxygenase (COX) included in the production of prostaglandins that are manufactured in answer to certain diseases or injury and cause pain, inflammation and swelling [2]. As well as other NSAIDs, naproxen was reported to be efficient in the prevention of many cancers [3-5]. Hydrazide-hydrazones [6-21] and 1,2,4-triazole rings [2226] have diverse biological activities.

In this study, 1,2,4-triazole containing hydrazide-hydrazones derived from naproxen have been synthesized. The purity of the synthesized compounds have been proven by thin layer chromatography (TLC) and high pressure liquid chromatography (HPLC), elementel analysis and melting point assay. Their structures have been characterized by ${ }^{1} \mathrm{H}-\mathrm{NMR},{ }^{13} \mathrm{C}-\mathrm{NMR}, \mathrm{HR}-\mathrm{MS}$ and FT-IR spectroscopic methods. The anticancer activity of the synthesized compounds were studied against PC-3, DU-143 and LNCaP prostate cancer cell lines.

How to cite this article: Han M, Bekçi H, Cumaoğlu A, Küçükgüzel ŞG. Synthesis and characterization of 1,2,4-triazole containing hydrazidehydrazones derived from (S)-Naproxen as anticancer agents. Marmara Pharm J. 2018; 22 (4): 559-569. 


\section{RESULTS AND DISCUSSION}

\subsection{Chemistry}

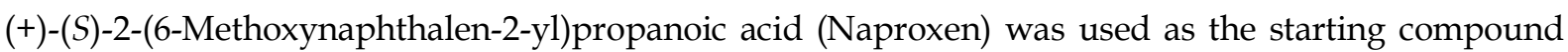
to design several new 1,2,4-triazole ring and hydrazide-hydrazones. Firstly, (S)-methyl 2-(6-methoxy-2naphthyl)propanoate (1) was prepared by the reaction of naproxen and methanol in the presence of a few drops of concentrated sulfuric acid. Compound $\mathbf{1}$ and hydrazine-hydrate $\% 80$ was refluxed in ethanol in order to prepare (S)-2-(6-methoxy-2-naphthyl)propanoic acid hydrazide (2). Equimolar 4fluorophenylisothiocyanate and compound 2 was heated in butanolic medium to prepare (S)-N-(4fluorophenyl)-2-[2-(6-methoxynaphtalen-2-yl)propanoyl]hydrazinecarbothioamide $\quad$ (3). (S)-5-[1-(6methoxynaphtalen-2-yl)ethyl]-4-fluorophenyl-4H-1,2,4-triazole-3-thione (4) was synthesized in $4 \mathrm{~N} \mathrm{NaOH}$ with reaction of compound 3 [31]. (S)-Ethyl \{[4-(4-fluorophenyl)-5-[(1-(6-methoxynaphtalen-2-yl)ethyl)]-4H1,2,4-triazole-3-yl]sulphanyl\}acetate (5) was prepared in solution of acetone with equimolar ethyl bromoacetate and twice as much mol of potassium carbonate. (S)-2-(\{5-[1-(6-Methoxynaphtalen-1-yl)ethyl]4-fluorophenyl-4H-1,2,4-triazole-3-yl\}sulphanyl)acetohydrazide (6) was synthesized with compound 5 and hydrazine-hydrate $(\% 80)$ in solution of ethanol. Our final compounds hydrazide-hydrazones (7a-m) was prepared with compound 6 and appropriate aromatic aldehydes in ethanolic medium in a few drops of glacial acetic acid. Compounds 5, 6 and 7a-m are original molecules in this study (Figure 1).

The structures of compounds 5, 6 and 7a-m were confirmed by elemental analysis, HPLC, TLC and spectral techniques such as ${ }^{1} \mathrm{H}-\mathrm{NMR},{ }^{13} \mathrm{C}-\mathrm{NMR}, \mathrm{HR}-\mathrm{MS}$ and FT-IR.

Optical rotation angle analysis has been performed to prove that Naproxen was S-enantiomer as reported in the raw material declaration certificate and in the literature [27]. In this study, optical rotation angle measurement was performed on a Rudolph Autopol V Plus brand / model polarimeter. For this measurement according to European Pharmacopoeia (EP) monograph [28], $0.5 \mathrm{~g}$ of Naproxen was dissolved in $25 \mathrm{ml}$ of pure ethanol. The measurement result $\left(+59.246^{\circ}\right)$ was in line with $(S)$-Naproxen's value which was reported by EP 7.0 as between $+59^{\circ}$ and $+62^{\circ}$ [28]. Therefore, we reported our target compounds as (S)isomers.

The purity of the synthesized compounds have been proven by TLC and HPLC. The purity of the compounds was controlled on TLC plates precoated with silica gel G in a solvent system comprising of petroleum ether: ethyl acetate (40:60, v/v) mixture as an eluent. The spots were located under UV light (254 $\mathrm{nm})\left(\mathrm{t}: 21^{\circ} \mathrm{C}\right)$. HPLC data of synthesized compounds 5, 6, 7a-m were performed on Agilent Technologies 1100 series using a reverse phase ZORBAX C8 column in gradient mode (Acetonitrile:double distilled water system was used as gradient system: 50:50 from 0 to $5 \mathrm{~min} ; 75: 25$ to 50:50 from 5 to $7 \mathrm{~min}$; 100:0 to 75:25 from 7 to $15 \mathrm{~min})$.

FT-IR spectral data of novel hydrazide-hydrazones 7a-m were observed hydrazone $\mathrm{N}-\mathrm{H}$, hydrazone $\mathrm{C}=\mathrm{O}$ and hydrazone $\mathrm{C}=\mathrm{N}$ streching data between 3080-3055, 1697-1674 and 1606-1602 $\mathrm{cm}^{-1}$, respectively. The streching data of $\mathrm{C}=\mathrm{O}$ were $1734 \mathrm{~cm}^{-1}$ for compound $5,1685 \mathrm{~cm}^{-1}$ for compound 6 , respectively.

In the ${ }^{1} \mathrm{H}-\mathrm{NMR}$ spectra of compound 5 , characteristic signals for $-\mathrm{CH}_{3}$ moiety have been detected 1.16 ppm and for $-\mathrm{CH}_{2}$ moiety $1.24 \mathrm{ppm}$ as signal protons. In the ${ }^{1} \mathrm{H}-\mathrm{NMR}$ spectra of compound 6 , characteristic signals for $-\mathrm{NH}-\mathrm{NH}_{2}$ moiety have been detected 9.31 and $4.32 \mathrm{ppm}$ as signal $-\mathrm{NH}$ and $-\mathrm{NH}_{2}$ protons respectively.

${ }^{1} \mathrm{H}-\mathrm{NMR}$ spectral data of compounds $\mathbf{7 a - m}$ showed supporting evidence to identify their structures. The two singlet signals belonging to azomethine proton in eight compounds $7 \mathbf{a}, 7 \mathbf{b}, 7 \mathbf{e}, 7 \mathbf{f}, 7 \mathbf{g}, 7 \mathbf{i}, 7 \mathbf{k}$ and $7 \mathbf{l}$ were detected at 7.93-8.38 ppm and 8.11- $8.57 \mathrm{ppm}$, compounds $7 \mathrm{c}$ and $7 \mathbf{j}$ were detected singlet azomethine proton peaks at 8.24 and 8.29 ppm respectively, compounds $7 \mathrm{~d}, 7 \mathrm{~h}$ and $7 \mathrm{~m}$ were detected azomethine proton at aromatic area. The -NH- proton of compounds 7a-m was detected two peaks in the range of 11.48-11.98 ppm and 11.57-12.13 ppm. Only compound $7 \mathrm{c}$ gave singlet peak at $11.89 \mathrm{ppm}$.

${ }^{13} \mathrm{C}-\mathrm{NMR}$ spectral data of compounds $7 \mathrm{a}-\mathrm{m}$ showed the signals belonging to $-\mathrm{C}=\mathrm{O}$ group and $-\mathrm{N}=\mathrm{CH}-$ group were detected at 169.01-168.24 ppm and 147.04-142.40 ppm, respectively. Thiomethylene carbon (-S$\left.\mathrm{CH}_{2}-\right)$ was screened at $34.85-34.57 \mathrm{ppm}$. In thioesther compound 5 alifatic carbons (-S- $\left.\mathrm{CH}_{2}-\mathrm{CO}-\mathrm{OCH}_{2}-\mathrm{CH}_{3}\right)$ detected at 61.21 and $13.92 \mathrm{ppm}$ respectively. These carbons disappeared in hydrazide compound $\mathbf{6}$.

It is thought that all the thirteen molecules hold half or one mole of ethanol in their structure. It was proven with elemental analysis, FT-IR and ${ }^{1} \mathrm{H}-\mathrm{NMR}$ spectrum. In FT-IR spectra O-H streching bands were screened at range of 3281-3201. In ${ }^{1} \mathrm{H}-\mathrm{NMR}$ spectra, $-\mathrm{CH}_{3}$ protons and $-\mathrm{CH}_{2}$ - protons were monitorized at 
the range of 0.9-1.3 ppm and 1.2-1.9 ppm, respectively. The -OH protons were observed at the range of 4.0$5.6 \mathrm{ppm}$.

The HR-MS spectra of six novel naproxen derivatives were studied in this study. The ionization format of $\mathbf{7 e}$ and $\mathbf{7 j}$ was electron impact (EI); the ionization format of compounds $\mathbf{5 , 6}, \mathbf{7 b}$ and $\mathbf{7 c}$ was electron spray impact (ESI). Compounds $\mathbf{7 e}$ and $\mathbf{7 j}$ gave the peak at their molecular weight. Other compounds gave the peak at $[\mathrm{M}+1]$ because of catching a hydrogen atom from medium. HR-MS spectrum of selected compound $7 \mathbf{e}$ displayed molecular ion peak at $\mathrm{m} / \mathrm{z} 379$. The major fragmentation pathway appeared by the cleavage of $-\mathrm{CH}_{2}-\mathrm{CONHN}=\mathrm{CH}-\mathrm{Ar}$ bonds of amide moiety (Figure 2 ).

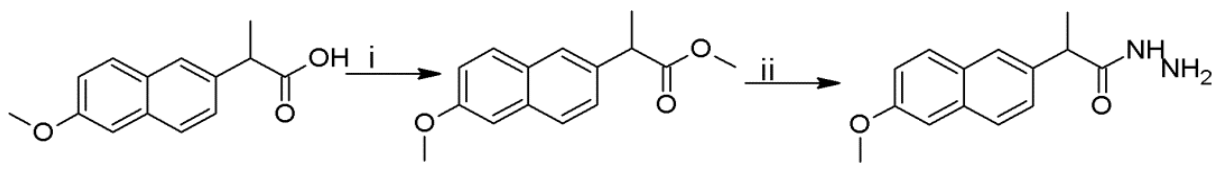<smiles>COc1cc2ccc(C(C)C(=O)NN)cc2cc1OC(C)=O</smiles>

(3)<smiles></smiles>

(5)<smiles>COc1ccc2cc(C(C)c3nnc(SCC(=O)N/C=C/[Ga])n3-c3ccc(F)cc3)ccc2c1</smiles>

(2)

(1)<smiles>COc1ccc2cc3c(cc2c1)C(C)C(C)N(c1ccc(F)cc1)c1n[nH]c(=S)n1-3</smiles>

(4)<smiles></smiles>

(6)

(7a-m)

Figure 1. Synthetic route to naproxen derivatives 7a-m.

i: $\mathrm{CH}_{3} \mathrm{OH} /$ d. $\mathrm{H}_{2} \mathrm{SO}_{4}$; ii: $\mathrm{NH}_{2} \mathrm{NH}_{2} \cdot \mathrm{H}_{2} \mathrm{O} / \mathrm{C}_{2} \mathrm{H}_{5} \mathrm{OH}$; iii: 4-F-C $\mathrm{C}_{4}-\mathrm{NCS} / n$-butanol; iv: $4 \mathrm{~N} \mathrm{NaOH}$; $v$ : $\mathrm{Br}_{2}-\mathrm{CH}_{2}-\mathrm{COOC}_{2} \mathrm{H}_{5} /$ $\mathrm{K}_{2} \mathrm{CO}_{3}$ / Acetone; vi: $\mathrm{NH}_{2} \mathrm{NH}_{2} \cdot \mathrm{H}_{2} \mathrm{O} / \mathrm{EtOH}$; vii: $\mathrm{EtOH} /$ g. $\mathrm{CH}_{3} \mathrm{COOH} / \mathrm{Ar}-\mathrm{CHO}$.
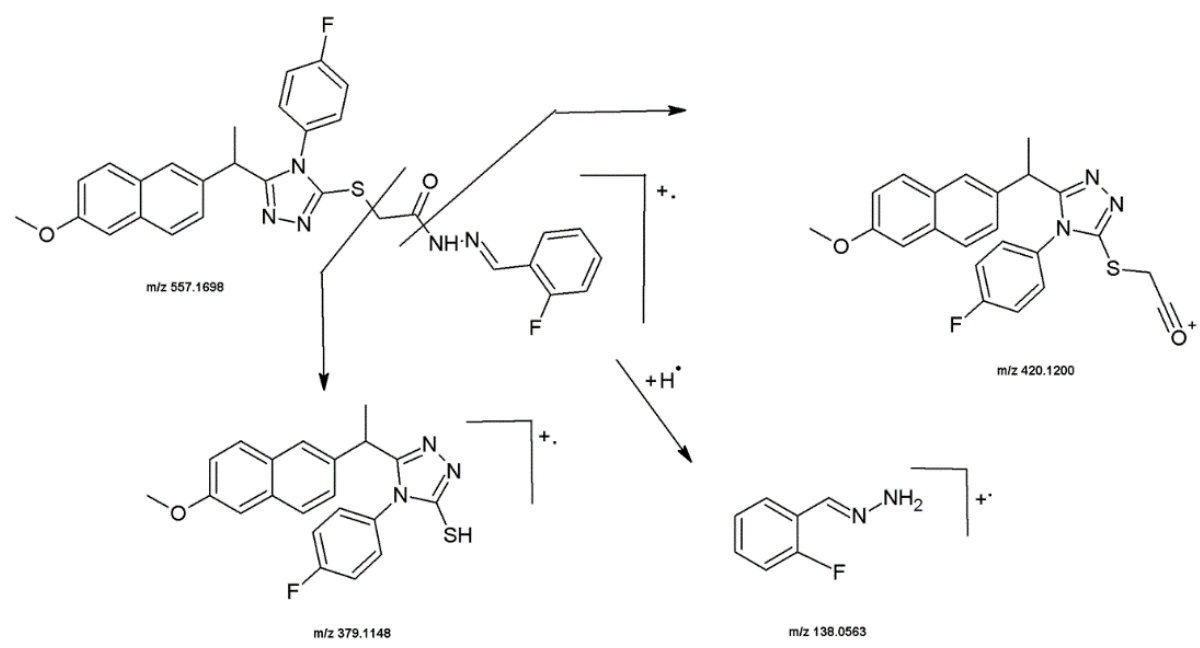

Figure 2. MS fragmentation pathway for the compound 7e. 


\subsection{Biological activity}

The anticancer activity of naproxen, the synthesis starting compound, has been reported. On the light of foregoing, we aimed to investigate the anticancer activity of (S)-naproxen hydrazide-hydrazones (7a-m). The anticancer activity of all compounds was evaluated in Faculty of Pharmacy, Erciyes University. The sensitivity of the proatate cancer cell lines towards the compounds was evaluated from MTS method [29]. The anticancer activity of the compounds were evaluated against three cancer cell lines. The cell lines used were PC-3 and DU-143 (androgen-independent human prostate cancer cell lines) and LNCaP (androgensensitive human prostate adenocarcinoma) prostate cancer cell lines. Cisplatin was used as the positive sensitivity reference standard for cell lines. Compounds $7 \mathrm{a}-\mathrm{m}$ were found with $\mathrm{IC}_{50}$ values of $87.2-400 \mu \mathrm{M}$ according to MTS assay. The results of anticancer activity of synthesized compounds $7 \mathbf{a}-\mathbf{m}$ are given Table 1 .

Table 1. $\mathrm{IC}_{50}$ values of compounds 7a-m.

\begin{tabular}{|c|c|c|c|c|c|}
\hline \multirow{2}{*}{ Compd. } & \multirow{2}{*}{ Lab Code } & \multirow{2}{*}{ A } & \multicolumn{3}{|c|}{$\mathrm{IC}_{50}(\mu \mathrm{M})$} \\
\hline & & & PC-3 & DU-145 & LNCaP \\
\hline Cisplatin & & & 39.9 & 9.6 & 20.7 \\
\hline $7 a$ & SGK-558 & 2-chloro-6-fluoro & $>400$ & $>400$ & $>400$ \\
\hline $7 \mathrm{~b}$ & SGK-560 & 2,4-dichloro & $400 \leq$ & $400 \leq$ & $400 \leq$ \\
\hline $7 \mathrm{c}$ & SGK-561 & 2,6-dichloro & $>400$ & $>400$ & $>400$ \\
\hline $7 d$ & SGK-563 & 3,4-dichloro & $400 \leq$ & 244,5 & $400 \leq$ \\
\hline $7 e$ & SGK-564 & 2-fluoro & $>400$ & $>400$ & $>400$ \\
\hline $7 f$ & SGK-567 & 2-chloro & $400 \leq$ & $400 \leq$ & $400 \leq$ \\
\hline $7 g$ & SGK-568 & 4-chloro & 115,1 & 87,2 & $>400$ \\
\hline $7 \mathrm{~h}$ & SGK-569 & 3-bromo & 170,0 & 97,71 & 263,3 \\
\hline $7 \mathbf{i}$ & SGK-570 & 4-bromo & $>400$ & 235,7 & $>400$ \\
\hline $7 \mathbf{j}$ & SGK-571 & 4-nitro & $>400$ & $>400$ & $>400$ \\
\hline $7 \mathbf{k}$ & SGK-573 & 4-methyl & $400 \leq$ & $400 \leq$ & 220,6 \\
\hline 71 & SGK-577 & 4-methoxy & $400 \leq$ & $400 \leq$ & $400 \leq$ \\
\hline $7 \mathrm{~m}$ & SGK-578 & 3,5-bis trifluoromethyl & 129,8 & 159,1 & $400 \leq$ \\
\hline
\end{tabular}

A computational study for prediction of ADME properties of the molecules was applied by determination of lipophilicity, topological polar surface area (TPSA), absorption (\% ABS) and simple molecular descriptors used by Lipinski in formulating his "rule of five". Calculations were performed using Molinspiration on-line property calculation toolkit (http://www.molinspiration.com) [30] (Table 2). Percentage of absorption (\% ABS) was estimated using the equation: \% ABS $=109-(0.345 \times$ TPSA $)$ [31].

Table 2. Predicted ADME, Lipinski parameters and molecular properties of the synthesized compounds 7a-m.

\begin{tabular}{lllllllll}
\hline Compound & $\%$ ABS & TPSA & n-ROTB & $\begin{array}{l}\text { n-ON } \\
\text { acceptors }\end{array}$ & $\begin{array}{l}\text { n-OHNH } \\
\text { donors }\end{array}$ & mi LogP & $\begin{array}{l}\text { Formula } \\
\text { Weight }\end{array}$ & $\begin{array}{l}\mathrm{n} \\
\text { violations }\end{array}$ \\
\hline $\mathbf{7 a}$ & 80 & 81.41 & 9 & 7 & 1 & 6.90 & 592.07 & 2 \\
$\mathbf{7 b}$ & 80 & 81.41 & 9 & 7 & 1 & 7.43 & 608.53 & 2 \\
$\mathbf{7 c}$ & 80 & 81.41 & 9 & 7 & 1 & 7.41 & 608.53 & 2 \\
$\mathbf{7 d}$ & 80 & 81.41 & 9 & 7 & 1 & 7.43 & 608.53 & 2 \\
$\mathbf{7 e}$ & 80 & 81.41 & 9 & 7 & 1 & 6.27 & 557.63 & 2 \\
$\mathbf{7 f}$ & 80 & 81.41 & 9 & 7 & 1 & 6.78 & 574.08 & 2 \\
$\mathbf{7 g}$ & 80 & 81.41 & 9 & 7 & 1 & 6.83 & 574.08 & 2 \\
$\mathbf{7 h}$ & 80 & 81.41 & 9 & 7 & 1 & 6.94 & 618.53 & 2 \\
$\mathbf{7 i}$ & 80 & 81.41 & 9 & 7 & 1 & 6.96 & 618.53 & 2 \\
$\mathbf{7 j}$ & 65 & 127.24 & 10 & 10 & 1 & 6.11 & 584.63 & 2 \\
$\mathbf{7 k}$ & 80 & 81.41 & 9 & 7 & 1 & 6.60 & 553.66 & 2 \\
$\mathbf{7 l}$ & 77 & 90.65 & 10 & 8 & 1 & 6.21 & 569.66 & 2 \\
$\mathbf{7 m}$ & 80 & 81.41 & 11 & 7 & 1 & 7.87 & 675.63 & 2 \\
Naproxen & 92 & 46.53 & 3 & 3 & 1 & 3.38 & 230.26 & 0 \\
\hline
\end{tabular}




\section{CONCLUSION}

Naproxen containing 1,2,4-triazole derivatives 5, 6 and 7a-m were synthesized and evaluated for their anticancer activity in this study. All of the compounds tested (compounds $7 \mathbf{a}-\mathbf{m}$ ) were found to be no or weak activity against PC-3, DU-145 and LNCaP cancer cell lines. Cisplatin was used as the positive sensitivity reference standard for cell lines.

\section{MATERIALS AND METHODS}

(S)-Naproxen was liberally ensured Abdi İbrahim. All aldehydes were purchased from Fluka and Aldrich. All other chemicals were purchased from Merck. Melting points were taken on Schmelzpunktbestimmer 9300 SMP II apparatus and are uncorrected. Synthesis of these compounds were carried out in Memmert WNB14 instrument and Heidolph MR Plug radley. Merck silica gel 60 F254 plates were used for analytical TLC. The purity of the compounds was controlled on TLC plates precoated with silica gel $G$ in a solvent system comprising of petroleum ether: ethyl acetate (40:60, v/v) mixture as an eluent. The spots were located under UV light $(254 \mathrm{~nm})\left(\mathrm{t}: 2 \mathrm{o}^{\circ} \mathrm{C}\right)$. HPLC data were performed on Agilent Technologies 1100 series. The separation was performed at ambient temperature using a reverse phase ZORBAX C8 column. All experiments were performed in gradient mode. Acetonitrile:bidistillee water system was used as gradient system: 50:50 from 0 to $5 \mathrm{~min} ; 75: 25$ to $50: 50$ from 5 to $7 \mathrm{~min}$; 100:0 to 75:25 from 7 to $15 \mathrm{~min}$; the flow rate was $1.0 \mathrm{~mL} / \mathrm{min}$ with monitoring at $254 \mathrm{~nm}$ (M1). Chromatographic data were collected and processed using Agilent Chemstation Plus software. Elemental analyses were performed on VarioMICRO V1.5.7. instrument. FT-IR spectra were run on Schimadzu FTIR-8400S spectrophotometer. ${ }^{1} \mathrm{H}-$ NMR and ${ }^{13}$ C-NMR spectra were obtained on a BRUKER AVANCE-DPX 300 instrument (Elementel analysis, ${ }^{1} \mathrm{H}-\mathrm{NMR}$ and ${ }^{13} \mathrm{C}-\mathrm{NMR}$ spectrum exist in İnönü University Scientific and Technological Research Center). MALDI-TOF HR-MS spectra using the EI and ESI ionization techniques were performed using a Jeol JMS-700 instrument. Optical rotation angle measurement for Naproxen was performed on a Rudolph Autopol V Plus brand / model polarimeter.

\subsection{Chemistry}

\subsubsection{Preparation of (S)-methyl 2-(6-methoxy-2-naphthyl)propanoate (1, CAS Number: 26159-35-3)}

In a few drops of concentrated sulfuric acid, Naproxen $(0.05 \mathrm{~mol})$ and methanol $(50 \mathrm{~mL})$ were refluxed for $8 \mathrm{~h}$. The contents of the flask were cooled and neutralized by using $\mathrm{NaHCO}_{3}(5 \%)$. The resulting precipitate was filtered, dried, and recrystallized from ethanol. Yield 75\%; M.p. $100-101{ }^{\circ} \mathrm{C}$ (lit $\left.88^{\circ} \mathrm{C}\right)$ [32, 33].

\subsubsection{Preparation of (S)-2-(6-methoxy-2-naphthyl)propanoic acid hydrazide (2, CAS Number: 57475-91-9)}

To a ethanolic solution of compound $(\mathbf{1})(20 \mathrm{~mL}, 0.01 \mathrm{~mol})$ was added hydrazine-hydrate $(80 \%, 20 \mathrm{~mL})$ and refluxed for $4 \mathrm{~h}$. The reaction mixture was then cooled, diluted with water and allowed to stand overnight. The precipitated solid was washed with water, dried, and recrystallized from ethanol. Yield 70\%; M.p. $137^{\circ} \mathrm{C}$ (lit 94) [33].

\subsubsection{Preparation of (S)-N-(4-fluorophenyl)-2-[2-(6-methoxynaphtalen-2-yl)propanoyl]hydrazinecarbothio amide (3,} CAS Number: 1003001-33-9)

A solution of compound (2) $(0.01 \mathrm{~mol})$ and equimolar amount of 4-fluorophenyl isothiocyanate in $n$ butanol $(20 \mathrm{~mL})$ was heated on radley for $4 \mathrm{~h}$. After the mixture was cooled at room temperature and $n$ butanol was evaporated. The product was dried and recrystallized with ethanol. Yield $75 \%$; M.p. $158-160{ }^{\circ} \mathrm{C}$ (lit $\left.162^{\circ} \mathrm{C}\right)$ [33].

4.1.4. Preparation of (S)-5-[1-(6-methoxynaphtalen-2-yl)ethyl]-4-fluorophenyl-4H-1,2,4-triazole-3-thione (4, CAS Number: 1003001-45-3)

A solution of compound (3) $(0.01 \mathrm{~mol})$ in $4 \mathrm{~N}$ sodium hydroxide solution $(30 \mathrm{~mL})$ was heated under radley for $16 \mathrm{~h}$. After cooling to room temperature, the solution was adjusted to $\mathrm{pH} 6$ by glacial acetic acid. The crude product was precipitated, filtered and washed with distilled water. The product was get by recrystallization from ethanol. Yield $60 \% ; 145-146^{\circ} \mathrm{C}$ (lit $\left.144^{\circ} \mathrm{C}\right)$ [33]. 


\subsubsection{Synthesis of (S)-ethyl\{[4-(4-fluorophenyl)-5-[(1-(6-methoxynaphtalen-2-yl)ethyl)]-4H-1,2,4-triazole-3-} yl]sulphanyljacetate (5)

Compound (4) $(0.001 \mathrm{~mol})$ was dissolved in $30 \mathrm{~mL}$ of acetone $(30 \mathrm{~mL})$ and potassium carbonate $(0.002$ $\mathrm{mol})$ was added to solution. Then, ethyl bromoacetate $(0.0011 \mathrm{~mol})$ were added to the solution to divide four times at 15 minutes intervals. The content of the flask was stirred at room temperature for $20 \mathrm{~h}$. The mixture was filtered of inorganic compounds. Then, aceton was evaporated and the precipitated compound was filtered, dried and recrystallized from methanol.

Yield: 84\%; Brown solid; M.p. 100-101 ${ }^{\circ} \mathrm{C}$; HPLC $\mathrm{t}_{\mathrm{R}}$ (min.): 8.07 (M1); FT-IR (vmax, $\left.\mathrm{cm}^{-1}\right)$ 2982, 2933, 1734 $(\mathrm{C}=\mathrm{O}), 1635,1508,1485,1448,1263,1211,1122$ (C-F); ${ }^{1} \mathrm{H}-\mathrm{NMR}\left(300 \mathrm{MHz}, \mathrm{DMSO}-d_{6}\right) \delta_{H}$ ppm: $1.16(\mathrm{t}, 3 \mathrm{H}$, $\left.\mathrm{C}_{3}\right), 1.24\left(\mathrm{q}, 2 \mathrm{H}, \underline{\mathrm{CH}}_{2}\right), 1.64\left(\mathrm{~d}, 3 \mathrm{H}, \mathrm{CH}-\underline{\mathrm{H}}_{3}\right), 3.85\left(\mathrm{~s}, 3 \mathrm{H}, \mathrm{OC}_{3}\right), 3.99\left(\mathrm{~s}, 2 \mathrm{H}, \mathrm{SC} \underline{\mathrm{H}}_{2}\right) 4.09$ (q, 1H, $\left.\underline{\mathrm{H}}_{-}-\mathrm{CH}_{3}\right)$, 7.01-7.65 (m, 10H, Ar- $\underline{\mathrm{H}})$; ${ }^{13} \mathrm{C}-\mathrm{NMR}\left(75 \mathrm{MHz}, \mathrm{DMSO}-d_{6}\right) \delta_{\mathrm{C}} \mathrm{ppm}: 168.04(\mathrm{C}=\mathrm{O}), 163.91,160.63,158.08,157.07$, $136.83,133.01,129.81,129.69,128.95,128.90,128.18,126.97,125.69,125.24,118.66,116.68,116.36,105.63,61.21$, 55.09, 36.09, 33.98, 20.67, 13.92; HR-MS (ESI) m/z: Calcd. for $\left[\mathrm{C}_{25} \mathrm{H}_{24} \mathrm{FN}_{3} \mathrm{O}_{3} \mathrm{~S} . \mathrm{H}^{+}\right] 466.1595$ found 466.1601; Anal. Calcd. for $\mathrm{C}_{25} \mathrm{H}_{24} \mathrm{FN}_{3} \mathrm{O}_{3} \mathrm{~S}$. $\mathrm{CH}_{3} \mathrm{OH}$ : C, 62.76, H, 5.67, N, 8.44, S, 6.44; found C, 62.28, H, 5.31, N, 8.31, S, 6.00 .

\subsubsection{Synthesis of (S)-2-(\{5-[1-(6-methoxynaphtalen-1-yl)ethyl]-4-fluorophenyl-4H-1,2,4-triazole-3-yl\}} sulphanyl)acetohydrazide (6)

To a mixture of compound (5) $(0.001 \mathrm{~mol})$ and hydrazine hydrate $(0.003 \mathrm{~mol}, \% 80)$, absolute ethanol $(50 \mathrm{~mL})$ was added and it was refluxed for $6 \mathrm{~h}$ on a water bath. The mixture was concentrated, cooled, and poured into cold water. It was kept for $24 \mathrm{~h}$ at fridge and the solid mass separated out was filtered, dried, and recrystallized from ethanol.

Yield: 75\%; White solid; M.p. 178-179 ${ }^{\circ} \mathrm{C}$; HPLC $\mathrm{t}_{\mathrm{R}}(\mathrm{min}$.$) : 6.37$ (M1); FT-IR ( $\left.\mathrm{v}_{\max }, \mathrm{cm}^{-1}\right) 3041(\mathrm{~N}-\mathrm{H})$, 3009, 2931, 1685 (C=O), 1508, 1489, 1448, 1259, 1220, 1126 (C-F); ${ }^{1} \mathrm{H}-\mathrm{NMR}$ (300 MHz, DMSO-d d $_{6} \delta_{H}$ ppm: 1.64

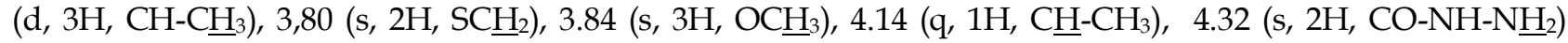

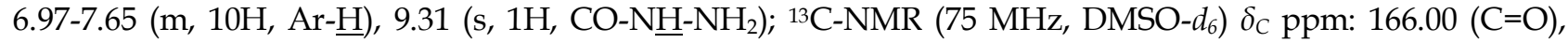
163.90, 160.35, 157.99, 157.06, 136.85, 133.01, 132.51, 129.89, 129.77, 128.98, 128.19, 126.97, 125.72, 125.24, 118.65, 116.64, 116.28, 105.63, 55.09, 36.10, 34.24, 20.69; HR-MS (ESI) m/z: Calcd. for $\left[\mathrm{C}_{23} \mathrm{H}_{22} \mathrm{FN}_{5} \mathrm{O}_{2} \mathrm{~S} . \mathrm{H}^{+}\right]$ 452.1551 found 452.1558; Anal. Calcd. for $\mathrm{C}_{23} \mathrm{H}_{22} \mathrm{FN}_{5} \mathrm{O}_{2} \mathrm{~S}$ : C, 61.18, $\mathrm{H}, 4.91, \mathrm{~N}, 15.51$; found $\mathrm{C}, 61.69, \mathrm{H}, 4.23$, $\mathrm{N}, 15.40$.

\subsubsection{General Procedure for the synthesis of (S)-2-\{[5-[1-(6-methoxynaphtalen-2-yl)ethyl]-4-(4-fluorophenyl)-4H-} 1,2,4-triazole-3-yl]sulphanyl\}-N'-[(substituted phenyl) methylidene]acetohydrazides (7a-m)

Compound (6) $(0.001 \mathrm{~mol})$ was dissolved in absolute ethanol. A few drop glacial acetic acid were added to solution. Equimolar amount of the appropriate aromatic aldehyde were added and refluxed for $8 \mathrm{~h}$. The flask content was allowed to cool, and the filtered and dried precipitate were recrystallized from ethanol.

\section{(S)-2-\{[5-[1-(6-Methoxynaphtalen-2-yl)ethyl]-4-(4-fluorophenyl)-4H-1,2,4-triazole-3-yl] sulphanyl\}-N'-[(2- chloro-6-fluorophenyl)methylidene]acetohydrazide (7a)}

Yield: 86\%; Pale brown solid; M.p. 152-154 ${ }^{\circ} \mathrm{C}$; HPLC $\mathrm{t}_{\mathrm{R}}$ (min.): 8.38 (M1); FT-IR (vmax, $\left.\mathrm{cm}^{-1}\right) 3064$ (NH), 2976, 2913, 1687 (C=O), 1604 (C=N), 1543, 1508, 1448, 1417, 1267, 1213, 1120 (C-F); ${ }^{1} \mathrm{H}-\mathrm{NMR}$ (300 MHz, DMSO- $\left.d_{6}\right) \delta_{H}$ ppm: $1.63\left(\mathrm{~d}, 3 \mathrm{H}, \mathrm{CH}-\underline{\mathrm{C}}_{3}\right), 3.84\left(\mathrm{~s}, 3 \mathrm{H}, \mathrm{OC} \underline{\mathrm{H}}_{3}\right), 4.14\left(\mathrm{q}, 1 \mathrm{H}, \mathrm{C} \underline{\mathrm{H}}-\mathrm{CH}_{3}\right) 3.99,4.33\left(2 \mathrm{~s}, 2 \mathrm{H}, \mathrm{SC} \underline{\mathrm{H}}_{2}\right)$, 7.01-7.65 (m, 13H, Ar- $\underline{\mathrm{H}})$ 8.23, 8.39 (2s, $1 \mathrm{H}, \mathrm{N}=\mathrm{C} \underline{\mathrm{H}}), 11.83,11.96(2 \mathrm{~s}, 1 \mathrm{H}, \mathrm{CO}-\mathrm{N} \underline{\mathrm{H}})$; ${ }^{13} \mathrm{C}-\mathrm{NMR}$ (75 MHz, DMSO$\left.d_{6}\right) \delta_{\mathrm{C}} \mathrm{ppm} ; 168.72(\mathrm{C}=\mathrm{O}), 163.50,160.60,157.94,157.05,150.18,146.87(\mathrm{C}=\mathrm{N}), 136.89,136.64,133.45,132.99$, 131.60, 129.86, 129.74, 129.06, 129.02, 128.18, 126.96, 126.33, 125.70, 125.23, 120.15, 118.63, 116.60, 116.28, 105.62, 55.08, 36.11, 34.57, 20.68; Anal. Calcd. for $\mathrm{C}_{30} \mathrm{H}_{24} \mathrm{ClF}_{2} \mathrm{~N}_{5} \mathrm{O}_{2} \mathrm{~S} .1 / 2 \mathrm{C}_{2} \mathrm{H}_{5} \mathrm{OH}$.; C, 60.86, H, 4.09, N, 11.83, S, 5.42; found C, 60.48, H, 4.38, N, 11.38, S, 5.20.

\section{(S)-2-\{[5-[1-(6-Methoxynaphtalen-2-yl)ethyl]-4-(4-fluorophenyl)-4H-1,2,4-triazole-3-yl]sulphanyl\}-N'-[(2,4-} dichlorophenyl)methylidene]acetohydrazide (7b)

Yield: 69\%; White solid; M.p. 118-120 ${ }^{\circ} \mathrm{C}$; HPLC $\mathrm{t}_{\mathrm{R}}(\mathrm{min}$.$) : 9.06$ (M1); FT-IR ( $\left.\mathrm{v}_{\max }, \mathrm{cm}^{-1}\right) 3078(\mathrm{~N}-\mathrm{H})$, 2997, 2935, $1680(\mathrm{C}=\mathrm{O}), 1604(\mathrm{C}=\mathrm{N}), 1550,1508,1485,1440,1265,1219,1118$ (C-F); ${ }^{1} \mathrm{H}-\mathrm{NMR}(300 \mathrm{MHz}$, DMSO- $\left.d_{6}\right) \delta_{H}$ ppm: $1.64\left(\mathrm{~d}, 3 \mathrm{H}, \mathrm{CH}-\underline{\mathrm{H}}_{3}\right), 3.84\left(\mathrm{~s}, 3 \mathrm{H}, \mathrm{OC}_{3}\right), 4.13\left(\mathrm{q}, 1 \mathrm{H}, \underline{\mathrm{C}} \underline{\mathrm{H}}-\mathrm{CH}_{3}\right), 3.99,4.40\left(2 \mathrm{~s}, 2 \mathrm{H}, \mathrm{SC} \underline{\mathrm{H}}_{2}\right)$,

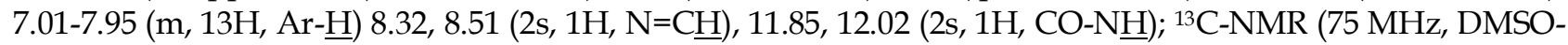


$\left.d_{6}\right) \delta_{\mathrm{C}}$ ppm; $168.73(\mathrm{C}=\mathrm{O}), 163.59,160.54,157.99,157.06,146.10(\mathrm{C}=\mathrm{N}), 138.70,136.87,133.82,133.65,133.00$, $131.55,130.27,129.88,129.76,129.37,128.96,128.18,127.94,126.96,125.69,125.24,118.64,116.59,116.29$, 105.64, 55.09, 36.11, 34.64, 20.68; HR-MS (ESI) m/z: Calcd. for $\left[\mathrm{C}_{30} \mathrm{H}_{24} \mathrm{Cl}_{2} \mathrm{FN}_{5} \mathrm{O}_{2} \mathrm{~S} . \mathrm{H}^{+}\right] 608.1084$ found 608.1096; Anal. Calcd. for $\mathrm{C}_{30} \mathrm{H}_{24} \mathrm{ClF}_{2} \mathrm{~N}_{5} \mathrm{O}_{2} \mathrm{~S} .1 / 2 \mathrm{H}_{2} \mathrm{O} .1 / 2 \mathrm{C}_{2} \mathrm{H}_{5} \mathrm{OH}$; C, 58.13, H, 4.41, N, 10.93, S, 5.01; found C, 57.59, $\mathrm{H}$, $3.88, \mathrm{~N}, 10.61, \mathrm{~S}, 4.59$.

(S)-2-\{[5-[1-(6-Methoxynaphtalen-2-yl)ethyl]-4-(4-fluorophenyl)-4H-1,2,4-triazole-3-yl]sulphanyl\}-N'-[(2,6dichlorophenyl)methylidene]acetohydrazide (7c)

Yield: 81\%; Cream colour solid; M.p. 125-127 ${ }^{\circ}$ C; HPLC $t_{R}$ (min.): 8.78 (M1); FT-IR ( vmax $\left._{\text {cm }} \mathrm{cm}^{-1}\right) 3055$ (NH), 2976, 2933, 1680 (C=O), 1604 (C=N), 1543, 1508, 1448, 1423, 1265, 1224, 1120 (C-F); ${ }^{1} \mathrm{H}-\mathrm{NMR}(300 \mathrm{MHz}$, DMSO- $\left.d_{6}\right) \delta_{H}$ ppm: $1.64\left(\mathrm{~d}, 3 \mathrm{H}, \mathrm{CH}-\underline{\mathrm{H}}_{3}\right), 3.84\left(\mathrm{~s}, 3 \mathrm{H}, \mathrm{OC}_{3}\right), 4.10\left(\mathrm{q}, 1 \mathrm{H}, \mathrm{C} \underline{\mathrm{H}}-\mathrm{CH}_{3}\right) 4.03,4.35$ (2s, 2H, SC 2 ), 7.01-7.65 (m, 13H, Ar- $\underline{\mathrm{H}}) 8.24(\mathrm{~s}, 1 \mathrm{H}, \mathrm{N}=\mathrm{C} \underline{\mathrm{H}}), 11.89(\mathrm{~s}, 1 \mathrm{H}, \mathrm{CO}-\mathrm{N} \underline{\mathrm{H}}) ;{ }^{13} \mathrm{C}-\mathrm{NMR}\left(75 \mathrm{MHz}, \mathrm{DMSO}-d_{6}\right) \delta_{\mathrm{C}} \mathrm{ppm} ;$ 168.82 (C=O), 163.56, 160.57, 157.93, 157.05, $143.98(\mathrm{C}=\mathrm{N}), 142.50,138.76,136.88,133.87,133.00,131.12,129.86$, $129.74,129.43,129.37,129.01,128.97,128.18,126.96,125.70,125.23,118.64,116.59,116.28,105.63,55.08,36.11$, 34.66, 20.68; HR-MS (ESI) m/z: Calcd. for $\left[\mathrm{C}_{30} \mathrm{H}_{24} \mathrm{Cl}_{2} \mathrm{FN}_{5} \mathrm{O}_{2} \mathrm{~S} . \mathrm{H}^{+}\right] 608.1084$ found 608.1099; Anal. Calcd. for $\mathrm{C}_{30} \mathrm{H}_{24} \mathrm{Cl}_{2} \mathrm{FN}_{5} \mathrm{O}_{2}$ S. $1 / 2 \mathrm{H}_{2} \mathrm{O} .1 / 2 \mathrm{C}_{2} \mathrm{H}_{5} \mathrm{OH}$; C, 58.13, H, 4.41, N, 10.93, S:5.01; found C, 57.59, H, 3.88, N, 10.61, S, 4.59 .

(S)-2-\{[5-[1-(6-Methoxynaphtalen-2-yl)ethyl]-4-(4-fluorophenyl)-4H-1,2,4-triazole-3-yl]sulphanyl\}-N'-[(3,4dichlorophenyl)methylidene]acetohydrazide (7d)

Yield: 84\%; White solid; M.p. 132-134 ${ }^{\circ} \mathrm{C}$; HPLC $\mathrm{t}_{\mathrm{R}}$ (min.): 8.55 (M1); FT-IR (vmax, $\left.\mathrm{cm}^{-1}\right) 3066$ (N-H), 2985, 2935, 1680 (C=O), $1604(\mathrm{C}=\mathrm{N}), 1550,1508,1483,1440,1265,1219,1120(\mathrm{C}-\mathrm{F}) ;{ }^{1} \mathrm{H}-\mathrm{NMR}(300 \mathrm{MHz}$, DMSO- $\left.d_{6}\right) \delta_{H}$ ppm: $1.64\left(\mathrm{~d}, 3 \mathrm{H}, \mathrm{CH}-\underline{\mathrm{H}}_{3}\right), 3.84\left(\mathrm{~s}, 3 \mathrm{H}, \mathrm{OCH}_{3}\right), 4.13\left(\mathrm{q}, 1 \mathrm{H}, \mathrm{CH}_{-} \mathrm{CH}_{3}\right), 4.00,4.40(2 \mathrm{~s}, 2 \mathrm{H}, \mathrm{SCH} 2)$, 7.01-8.15 (m, 13H, Ar- $\underline{\mathrm{H}} ; \mathrm{s}, 1 \mathrm{H}, \mathrm{N}=\mathrm{C} \underline{\mathrm{H}}), 11.79,11.92(2 \mathrm{~s}, 1 \mathrm{H}, \mathrm{CO}-\mathrm{N} \underline{\mathrm{H}}) ;{ }^{13} \mathrm{C}-\mathrm{NMR}\left(75 \mathrm{MHz}, \mathrm{DMSO}-d_{6}\right) \delta_{\mathrm{C}}$ ppm; $168.76(\mathrm{C}=\mathrm{O}), 163,62,160.59,157.97,157.06,144.32(\mathrm{C}=\mathrm{N}), 141.09,136.89,134.75,133.00,132.10,131.69,131.02$, $129.88,129.76,128.96,128.18,126.96,126.79,126.71,125.69,125.23,118.64,116.61,116.29,105.63,55.08,36.12$, 34.65, 20.68; Anal. Calcd. for $\mathrm{C}_{30} \mathrm{H}_{24} \mathrm{Cl}_{2} \mathrm{FN}_{5} \mathrm{O}_{2} \mathrm{~S}$. $\mathrm{C}_{2} \mathrm{H}_{5} \mathrm{OH}$; C, 58.72, H, 4.62, N, 10.70, S, 4.90; found C, 58.35, $\mathrm{H}$, $3.99, \mathrm{~N}, 10.66, \mathrm{~S}, 4.82$.

(S)-2-\{[5-[1-(6-Methoxynaphtalen-2-yl)ethyl]-4-(4-fluorophenyl)-4H-1,2,4-triazole-3-yl]sulphanyl\}-N'-[(2fluorophenyl)methylidene]acetohydrazide (7e)

Yield: 69\%; White solid; M.p. 124-127 ${ }^{\circ} \mathrm{C}$; HPLC $\mathrm{t}_{\mathrm{R}}$ (min.): 7.88 (M1); FT-IR (vmax, $\left.\mathrm{cm}^{-1}\right) 3064$ (N-H), 2976, 2933, 1680 (C=O), 1604 (C=N), 1564, 1550, 1508, 1490, 1265, 1230, 1136 (C-F); ${ }^{1} \mathrm{H}-\mathrm{NMR}(300 \mathrm{MHz}$, DMSO- $\left.d_{6}\right) \delta_{H}$ ppm: $1.63\left(\mathrm{~d}, 3 \mathrm{H}, \mathrm{CH}-\underline{\mathrm{H}}_{3}\right), 3.84\left(\mathrm{~s}, 3 \mathrm{H}, \mathrm{OC}_{3}\right), 4.14\left(\mathrm{q}, 1 \mathrm{H}, \underline{\mathrm{CH}}_{-}-\mathrm{CH}_{3}\right) 3.99,4.40\left(2 \mathrm{~s}, 2 \mathrm{H}, \mathrm{SC} \underline{\mathrm{H}}_{2}\right)$,

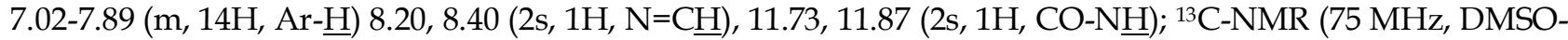
$\left.d_{6}\right) \delta_{\mathrm{C}} \mathrm{ppm} ; 168.62(\mathrm{C}=\mathrm{O}), 163.40,160.62,158.97,157.96,157.06,146.67(\mathrm{C}=\mathrm{N}), 136.90,136.57,133.00,131.95$, $129.89,129.77,128.97,128.19,126.95,126.19,125.70,125.24$, , 121.39, 118.64, 116.61, 116.29, 116.15, 115.87, 105.64, 55.09, 36.13, 34.66, 20.70; HR-MS (EI) m/z: Calcd. for $\left[\mathrm{C}_{30} \mathrm{H}_{25} \mathrm{~F}_{2} \mathrm{~N}_{5} \mathrm{O}_{2} \mathrm{~S}\right]$ 557.1697 found 557.1698; Anal. Calcd. for $\mathrm{C}_{30} \mathrm{H}_{25} \mathrm{~F}_{2} \mathrm{~N}_{5} \mathrm{O}_{2}$ S. $1 / 2 \mathrm{C}_{2} \mathrm{H}_{5} \mathrm{OH}$; C, 64.07, H, 4.82, N, 12.05, S, 5.34; found C, 63.98, H, 5.07, N, 12.01, S, 5.33 .

(S)-2-\{[5-[1-(6-Methoxynaphtalen-2-yl)ethyl]-4-(4-fluorophenyl)-4H-1,2,4-triazole-3-yl]sulphanyl\}-N'-[(2chlorophenyl)methylidene]acetohydrazide (7f)

Yield: 75\%; White solid; M.p. 152-155 oC; HPLC tR (min.): 8.29 (M1); FT-IR (vmax, cm $\left.{ }^{-1}\right) 3072$ (N-H), 2997, 2935, 1680 (C=O), 1604 (C=N), 1543, 1508, 1485, 1435, 1265, 1219, 1116 (C-F); $1 \mathrm{H}-\mathrm{NMR}(300 \mathrm{MHz}$, DMSO- $\left.d_{6}\right) \delta_{H}$ ppm: $1.63\left(\mathrm{~d}, 3 \mathrm{H}, \mathrm{CH}-\underline{\mathrm{H}}_{3}\right), 3.84\left(\mathrm{~s}, 3 \mathrm{H}, \mathrm{OCH}_{3}\right), 4.14\left(\mathrm{q}, 1 \mathrm{H}, \underline{\mathrm{CH}}_{-} \mathrm{CH}_{3}\right), 3.98,4.41(2 \mathrm{~s}, 2 \mathrm{H}, \mathrm{SCH} 2)$, 7.07-7.95 (m, 14H, Ar- $\underline{\mathrm{H}})$ 8.38, $8.57(2 \mathrm{~s}, 1 \mathrm{H}, \mathrm{N}=\mathrm{CH}), 11.81,11.98$ (2s, 1H, CO-NH); $13 \mathrm{C}-\mathrm{NMR}$ (75 MHz, DMSO$\left.d_{6}\right) \delta_{\mathrm{C}} \mathrm{ppm} ; 168.68(\mathrm{C}=\mathrm{O}), 163.49,160.59,157.96,157.06,142.87(\mathrm{C}=\mathrm{N}), 139.71,136.90,133.13,133.00,131.61$, $131.40,131.15,129.90,129.77,129.10,128.97,128.19,127.65,126.96,125.70,125.24,118.64,116.59,116.29$, 105.63, 55.09, 36.12, 34.70, 20.70; Anal. calcd. for $\mathrm{C}_{30} \mathrm{H}_{25} \mathrm{ClFN}_{5} \mathrm{O}_{2} \mathrm{~S} . \mathrm{C}_{2} \mathrm{H}_{5} \mathrm{OH}$; C, 61.98, H, 5.04, N, 11.29, S, 5.17; found C, 61.08, H, 4.24, N, 10.76, S, 4.81 .

(S)-2-\{[5-[1-(6-Methoxynaphtalen-2-yl)ethyl]-4-(4-fluorophenyl)-4H-1,2,4-triazole-3-yl] sulphanyl\}-N'-[(4chlorophenyl)methylidene]acetohydrazide (7g)

Yield: 58\%; White solid; M.p. 141-142 ${ }^{\circ} \mathrm{C} ; \mathrm{HPLC} \mathrm{t}_{\mathrm{R}}(\mathrm{min}):$.8.03 (M1); FT-IR (vmax, $\left.\mathrm{cm}^{-1}\right) 3057$ (N-H), 2976, 2902, 1697 (C=O), 1606 (C=N), 1543, 1508, 1490, 1417, 1271, 1213, 1134 (C-F); ${ }^{1} \mathrm{H}-\mathrm{NMR}(300 \mathrm{MHz}$, 


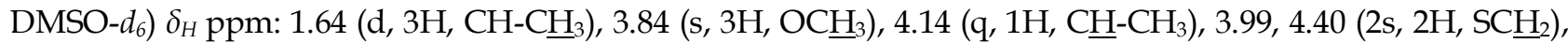
7.01-7.73 (m, 14H, Ar- $\underline{\mathrm{H}})$ 7.98, 8.17 (2s, 1H, N=CH), 11.70, 11.81 (2s, 1H, CO-NH); $13 \mathrm{C}-\mathrm{NMR}$ (75 MHz, DMSO$\left.d_{6}\right) \delta_{\mathrm{C}} \mathrm{ppm} ; 168.58(\mathrm{C}=\mathrm{O}), 163.40,160.59,157.94,157.06,142.40(\mathrm{C}=\mathrm{N}), 136.90,134.37,133.00,132.86,131.13$, $129.89,129.77,128.97,128.89,128.73,128.46,128.19,127.62,126.95,125.71,125.24,118.64,116.60,116.28$, 105.63, 55.08, 36.12, 34.75, 20.71; Anal. Calcd. for $\mathrm{C}_{30} \mathrm{H}_{25} \mathrm{ClFN}_{5} \mathrm{O}_{2} \mathrm{~S}$. $\mathrm{C}_{2} \mathrm{H}_{5} \mathrm{OH} ; \mathrm{C}, 61.98, \mathrm{H}, 5.04, \mathrm{~N}, 11.29$, S, 5.17; found C, 62.69, H, 4.42, N, 11.36, S, 4.68.

(S)-2-\{[5-[1-(6-Methoxynaphtalen-2-yl)ethyl]-4-(4-fluorophenyl)-4H-1,2,4-triazole-3-yl]sulphanyl\}-N'-[(3bromophenyl)methylidene]acetohydrazide (7h)

Yield: 79\%; White solid; M.p. 170-172 ${ }^{\circ}$; HPLC $t_{R}$ (min.): 8.55 (M1); FT-IR (vmax, cm $\left.{ }^{-1}\right) 3066$ (N-H), 2985, 2933, 1680 (C=O), 1604 (C=N), 1558, 1508, 1483, 1438, 1263,1211, 1120 (C-F); ${ }^{1} \mathrm{H}-\mathrm{NMR}(300 \mathrm{MHz}$, DMSO-d $d_{6} \delta_{H}$ ppm: $1.64\left(\mathrm{~d}, 3 \mathrm{H}, \mathrm{CH}-\underline{\mathrm{CH}}_{3}\right), 3.84\left(\mathrm{~s}, 3 \mathrm{H}, \mathrm{OCH}_{3}\right), 4.14\left(\mathrm{q}, 1 \mathrm{H}, \mathrm{CH}_{-} \mathrm{CH}_{3}\right), 3.98,4.39(2 \mathrm{~s}, 2 \mathrm{H}, \mathrm{SCH} 2)$, 7.02-8.15 (m, 14H, Ar- $\underline{\mathrm{H}}, \mathrm{s}, 1 \mathrm{H}, \mathrm{N}=\mathrm{C} \underline{\mathrm{H}}), 11.71,11.84(2 \mathrm{~s}, 1 \mathrm{H}, \mathrm{CO}-\mathrm{N} \underline{\mathrm{H}})$; ${ }^{13 \mathrm{C}-\mathrm{NMR}}\left(75 \mathrm{MHz}, \mathrm{DMSO}-d_{6}\right) \delta_{\mathrm{C}} \mathrm{ppm}$; 168.70 (C=O), 163.43, 160.60, 157.98, 157.06, $145.27(\mathrm{C}=\mathrm{N}), 141.98,136.89,136.36,133.00,131.69,130.96,130.85$, $129.89,129.76,128.97,128.18,126.97,125.69,125.23,122.17,119.88,118.65,116.61,116.29,105.63,55.09,36.11$, 34.69, 20.68; Anal. Calcd. for $\mathrm{C}_{30} \mathrm{H}_{25} \mathrm{BrFN}_{5} \mathrm{O}_{2} \mathrm{~S} . \mathrm{H}_{2} \mathrm{O} . \mathrm{C}_{2} \mathrm{H}_{5} \mathrm{OH}$; C, 56.31, H, 4.87, N, 10.26, S, 4.70; found C, $56.12, \mathrm{H}, 3.96, \mathrm{~N}, 10.05, \mathrm{~S}, 4.56$.

(S)-2-\{[5-[1-(6-Methoxynaphtalen-2-yl)ethyl]-4-(4-fluorophenyl)-4H-1,2,4-triazole-3-yl]sulphanyl\}-N'-[(4bromophenyl)methylidene]acetohydrazide (7i)

Yield: 86\%; Pale brown solid; M.p. 133-135 ${ }^{\circ}$ C; HPLC $t_{R}(\min ):$.8.57 (M1); FT-IR (vmax, $\left.\mathrm{cm}^{-1}\right) 3057$ (N$\mathrm{H})$, 2974, 2933, 1687 (C=O), 1604 (C=N), 1543, 1508, 1485, 1448, 1267, 1220, 1120 (C-F); ${ }^{1} \mathrm{H}-\mathrm{NMR}(300 \mathrm{MHz}$, DMSO-d $\delta_{6} \delta_{H}$ ppm: $1.64\left(\mathrm{~d}, 3 \mathrm{H}, \mathrm{CH}-\underline{\mathrm{CH}}_{3}\right), 3.84\left(\mathrm{~s}, 3 \mathrm{H}, \mathrm{OCH}_{3}\right), 4.14\left(\mathrm{q}, 1 \mathrm{H}, \mathrm{CH}_{-}-\mathrm{CH}_{3}\right), 3.99,4.37(2 \mathrm{~s}, 2 \mathrm{H}, \mathrm{SCH} 2)$, 7.01-7.84 (m, 14H, Ar- $\underline{\mathrm{H}})$ 7.96, 8.15 (2s, 1H, N=CH), 11.70, 11.81 (2s, 1H, CO-NH); ${ }^{13} \mathrm{C}-\mathrm{NMR}$ (75 MHz, DMSO$\left.d_{6}\right) \delta_{\mathrm{C}} \mathrm{ppm} ; 168.59(\mathrm{C}=\mathrm{O}), 163.43,160.59,158.05,157.69,157.06,145.76(\mathrm{C}=\mathrm{N}), 142.54,136.88,136.85,133.18$, $133.00,131.79,129.89,129.76,128.96,128.69,128.18,126.97,125.69,125.24,123.17,118.65,116.61,116.29$, 105.63, 55.09, 36.12, 34.74, 20.68; Anal. Calcd. for $\mathrm{C}_{30} \mathrm{H}_{24} \mathrm{BrFN}_{5} \mathrm{O}_{2} \mathrm{~S}$. $\mathrm{C}_{2} \mathrm{H}_{5} \mathrm{OH}$; C, 57.83, H, 4.70, N, 10.54, S,4.82; found C, 57.38, H, 4.03, N, 10.68, S, 4.18.

(S)-2-\{[5-[1-(6-Metoxynaphtalen-2-yl)ethyl]-4-(4-fluorophenyl)-4H-1,2,4-triazole-3-yl]sulphanyl\}-N'-[(4nitrophenyl)methylidene]acetohydrazide (7j)

Yield: 77\%; Orange solid; M.p. 167-170 ${ }^{\circ} \mathrm{C}$; HPLC $\mathrm{t}_{\mathrm{R}}$ (min.): 7.78 (M1); FT-IR (vmax, $\left.\mathrm{cm}^{-1}\right) 3057$ (N-H), 2958, 2931, 1691 (C=O), 1602 (C=N), 1572, 1508, 1483, 1438, 1265, 1209, 1134 (C-F); ${ }^{1} \mathrm{H}-\mathrm{NMR}(300 \mathrm{MHz}$,

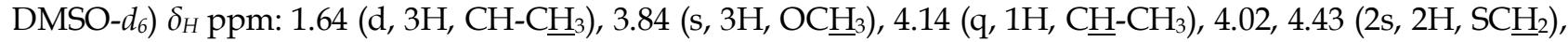
7.02-7.97 (m, 14H, Ar- $\underline{\mathrm{H}}) 8.29(\mathrm{~s}, 1 \mathrm{H}, \mathrm{N}=\mathrm{C} \underline{\mathrm{H}}), 11.92,12.03(2 \mathrm{~s}, 1 \mathrm{H}, \mathrm{CO}-\mathrm{NH})$; ${ }^{13} \mathrm{C}-\mathrm{NMR}\left(75 \mathrm{MHz}, \mathrm{DMSO}-d_{6}\right) \delta_{\mathrm{C}}$ ppm; 168.93 (C=O), 163.82, 160.57, 157.99, 157.06, 147.73, $144.50(\mathrm{C}=\mathrm{N}), 141.36,140.37,140.18,136.88,133.00$, 129.89, 129.77, 128.97, 128.18, 128.03, 127.76, 126.96, 125.70, 125.24, 124.03, 118.65, 116.61, 116.31, 105.62, 55.08, 36.11, 34.62, 20.70; HR-MS (EI) m/z: Calcd. for $\left[\mathrm{C}_{30} \mathrm{H}_{25} \mathrm{FN}_{6} \mathrm{O}_{4} \mathrm{~S}\right] 584.1642$ found 584.1630; anal. calcd. for $\mathrm{C}_{30} \mathrm{H}_{25} \mathrm{FN}_{6} \mathrm{O}_{4}$ S. $1 / 2 \mathrm{C}_{2} \mathrm{H}_{5} \mathrm{OH}$; C, 61.28, H, 4.61, N, 13.83, S, 5.27; found C, 60.28, H, 4.21, N, 13.70, S, 5.16.

(S)-2-\{[5-[1-(6-Methoxynaphtalen-2-yl)ethyl]-4-(4-fluorophenyl)-4H-1,2,4-triazole-3-yl] sulphanyl\}-N'-[(4methylphenyl)methylidene]acetohydrazide (7k)

Yield: 76\%; White solid; M.p. 120-122 ${ }^{\circ} \mathrm{C} . \mathrm{HPLC} \mathrm{t}_{\mathrm{R}}$ (min.): 8.23 (M1); FT- IR (vmax, $\mathrm{cm}^{-1}$ ) 3078 (N-H), 2985, 2935, $1674(\mathrm{C}=\mathrm{O}), 1604(\mathrm{C}=\mathrm{N}), 1554,1508,1483,1442,1265,1224,1120$ (C-F); ${ }^{1} \mathrm{H}-\mathrm{NMR}(300 \mathrm{MHz}$, DMSO-d $\delta_{6} \delta_{H}$ ppm: $1.64\left(\mathrm{~d}, 3 \mathrm{H}, \mathrm{CH}-\underline{\mathrm{H}}_{3}\right), 3.84\left(\mathrm{~s}, 3 \mathrm{H}, \mathrm{OCH}_{3}\right), 4.13\left(\mathrm{q}, 1 \mathrm{H}, \underline{\mathrm{CH}}_{-} \mathrm{CH}_{3}\right), 3.97,4.38\left(2 \mathrm{~s}, 2 \mathrm{H}, \mathrm{SC} \underline{\mathrm{H}}_{2}\right)$,

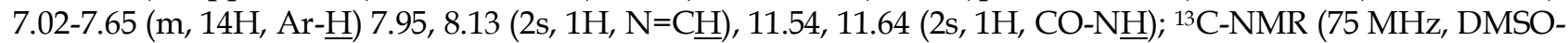
$\left.\mathrm{d}_{6}\right) \delta_{\mathrm{C}} \mathrm{ppm} ; 168.38(\mathrm{C}=\mathrm{O}), 163.18,160.59,158.04,157.94,157.06,147.04(\mathrm{C}=\mathrm{N}), 143.82,139.99,139.78,136.90$, $133.00,131.27,129.89,129.77,129.40,129.06,128.98,128.19,126.97,125.70,125.23,118.64,116.58,116.29$, 105.63, 55.08, 36.12, 35.08, 34.79, 20.69; Anal. Calcd. for $\mathrm{C}_{31} \mathrm{H}_{28} \mathrm{FN}_{5} \mathrm{O}_{2} \mathrm{~S} .1 / 2 \mathrm{C}_{2} \mathrm{H}_{5} \mathrm{OH}$; C, 66.65, H, 5.42, N, 12.14, S, 5.56; found C, 66.97, H, 5.00, N, 11.68, S, 5.21.

(S)-2-\{[5-[1-(6-Methoxynaphtalen-2-yl)ethyl]-4-(4-fluorophenyl)-4H-1,2,4-triazole-3-yl]sulphanyl\}-N'-[(4methoxyphenyl)methylidene]acetohydrazide (71)

Yield: 74\%; White solid; M.p. 128-130 ${ }^{\circ} \mathrm{C} . \mathrm{HPLC} \mathrm{t}_{\mathrm{R}}$ (min.): 7.63 (M1); FT-IR (vmax, $\left.\mathrm{cm}^{-1}\right) 3066$ (N-H), 2974, 2933, $1674(\mathrm{C}=\mathrm{O}), 1602(\mathrm{C}=\mathrm{N}), 1573,1508,1485,1440,1249,1222,1120(\mathrm{C}-\mathrm{F})$; ${ }^{1} \mathrm{H}-\mathrm{NMR}(300 \mathrm{MHz}$, DMSO-d $)_{6} \delta_{H}$ ppm: $1.64\left(\mathrm{~d}, 3 \mathrm{H}, \mathrm{CH}-\underline{\mathrm{H}}_{3}\right), 3.80\left(\mathrm{~s}, 3 \mathrm{H}, \mathrm{OC}_{3}\right), 3.84\left(\mathrm{~s}, 3 \mathrm{H}, \mathrm{OC}_{3}\right), 4.14\left(\mathrm{q}, 1 \mathrm{H}, \mathrm{C}_{\underline{H}}-\mathrm{CH}_{3}\right), 3.96$, 
$4.37\left(2 \mathrm{~s}, 2 \mathrm{H}, \mathrm{SC}_{2}\right), 6.98-7.64(\mathrm{~m}, 14 \mathrm{H}, \mathrm{Ar}-\underline{\mathrm{H}}) 7.93,8.11(2 \mathrm{~s}, 1 \mathrm{H}, \mathrm{N}=\mathrm{C} \underline{\mathrm{H}}), 11.48,11.57(2 \mathrm{~s}, 1 \mathrm{H}, \mathrm{CO}-\mathrm{N} \underline{\mathrm{H}}) ;{ }^{13} \mathrm{C}-$ NMR $\left(75 \mathrm{MHz}, \mathrm{DMSO}-\mathrm{d}_{6}\right) \delta_{\mathrm{C}} \mathrm{ppm} ; 168.24(\mathrm{C}=\mathrm{O}), 163.01,160.70,158.03,157.93,157.05,150.11,146.88(\mathrm{C}=\mathrm{N})$, $143.59,136.90,132.99,129.89,129.77,129.11,129.07,128.97,128.18,126.96,125.70,125.23,118.64,116.58$, $116.28,114.48,114.28,105.63,55.26,55.08,36.13,34.82$, 20.69; Anal. Calcd. for $\mathrm{C}_{31} \mathrm{H}_{28} \mathrm{FN}_{5} \mathrm{O}_{3} \mathrm{~S} .{ }^{1}{ }_{2} \mathrm{C}_{2} \mathrm{H}_{5} \mathrm{OH}$; C, 64.85, H, 5.27, N, 11.82, S, 5.41; found C, 64.71, H, 4.84, N, 11.25, S, 5.15.

(S)-2-\{[5-[1-(6-Methoxynaphtalen-2-yl)ethyl]-4-(4-fluorophenyl)-4H-1,2,4-triazole-3-yl]sulphanyl\}-N'-[(3,5bistrifluoromethylphenyl)methylidene]acetohydrazide $(7 \mathrm{~m})$

Yield: 42\%; White solid; M.p. 138-139 ${ }^{\circ} \mathrm{C}$; HPLC tR (min.): 9.18 (M1); FT-IR ( $\left.\mathrm{v}_{\max }, \mathrm{cm}^{-1}\right) 3080(\mathrm{~N}-\mathrm{H})$, 2989, 2932, 1681 (C=O), 1604 (C=N), 1543, 1508, 1485, 1442, 1276, 1211, 1132 (C-F); ${ }^{1} \mathrm{H}-\mathrm{NMR}$ (300 MHz, DMSO- $\left.d_{6}\right) \delta_{H}$ ppm: $1.63\left(\mathrm{~d}, 3 \mathrm{H}, \mathrm{CH}-\underline{\mathrm{H}}_{3}\right), 3.84\left(\mathrm{~s}, 3 \mathrm{H}, \mathrm{OC} \underline{\mathrm{H}}_{3}\right), 4.14\left(\mathrm{q}, 1 \mathrm{H}, \mathrm{C}_{-}-\mathrm{CH}_{3}\right), 4.04,4.44\left(2 \mathrm{~s}, 2 \mathrm{H}, \mathrm{SC} \underline{\mathrm{H}}_{2}\right)$, 7.01-8.35 (m, 13H, Ar- $\underline{\mathrm{H}}$; s, $1 \mathrm{H}, \mathrm{N}=\mathrm{C} \underline{\mathrm{H}}), 11.98,12.13(2 \mathrm{~s}, 1 \mathrm{H}, \mathrm{CO}-\mathrm{N} \underline{\mathrm{H}}) ;{ }^{13} \mathrm{C}-\mathrm{NMR}\left(75 \mathrm{MHz}, \mathrm{DMSO}-d_{6}\right) \delta_{\mathrm{C}} \mathrm{ppm}$; $169.01(\mathrm{C}=\mathrm{O}), 163.96,160.57,158.08,157.06,145.97(\mathrm{C}=\mathrm{N}), 140.54,136.88,136.78,133.00,131.38,130.97,130.59$, $129.87,129.74,129.39,128.96,128.18,127.21,127.06,126.97,126.21,125.68,125.23,118.64,116.64,116.25$, 105.62, 55.08, 36.10, 34.85, 20.66; Anal. Calcd. for $\mathrm{C}_{32} \mathrm{H}_{24} \mathrm{~F}_{7} \mathrm{~N}_{5} \mathrm{O}_{2} \mathrm{~S} .{ }^{1 / 2} \mathrm{C}_{2} \mathrm{H}_{5} \mathrm{OH}$; C, 56.73, H, 3.90, N, 10.02, S, 4.59; found C, 56.44, H, 3.61, N, 10.36, S, 4.86 .

\subsection{Anticancer Activity}

The anticancer activity of all compounds were evaluated in Faculty of Pharmacy, Erciyes University. The sensitivity of the cell lines towards the compounds was evaluated from MTS method. The anticancer activity of the compounds were evaluated against three cancer cell lines. The cell lines used were PC-3, DU145 and LNCaP. Anticancer activities of the compounds tested against that cell lines based on MTS assay [29]. Cell proliferative activity was measured using the 3-(4,5-dimethylthiazol-2-yl)-5-(3carboxymethoxyphenyl)-2-(4-sulfophenyl)-2H-tetrazolium (MTS) assay kit (Promega, Madison, WI, USA). Cisplatin was used as the positive sensitivity reference standard for cell lines.

Acknowledgements: This work was supported by The Scientific and Technical Research Council of Turkey (TÜBITAK), Research Fund Project Number: 215S009. The authors are grateful to Jürgen Gross from the Institute of Organic Chemistry, University of Heidelberg, for his generous help in obtaining HR-MS mass spectra of the synthesized compounds.

Author contributions: Concept - M.İ.H., Ş.G.K.; Design - M.İ.H., Ş.G.K.; Supervision - M.İ.H., Ş.G.K.; Resource M.İ.H., Ş.G.K.; Materials - M.İ.H., Ş.G.K., H.B., A.C.; Data collection and/or processing - M.İ.H., Ş.G.K., H.B., A.C.; Analysis and/or Interpretation - M.İ.H., Ş.G.K., H.B., A.C.; Literature Search - M.İ.H., Ş.G.K., H.B., A.C.; Writing M.İ.H., Ş.G.K.; Critical Reviews - M.İ.H., Ş.G.K., H.B., A.C.

Conflict of interest statement: The authors declare no conflict of interest.

\section{REFERENCES}

[1] Hawkey CJ. COX-2 inhibitors. The Lancet 1999; 353: 307-314.

[2] Ammar YA, Salem MA, Fayed EA, Helal MH, El-Gaby MSA, Thabet HK. Naproxen derivatives: Synthesis, reactions, and biological applications. Synth Commun. 2017; 47; 1341-1367. [CrossRef]

[3] Harris RE, Donk JB, Alshafie GA. Similar reductions in the risk of human colon cancer by selective and nonselective cyclooxygenase-2 (COX-2) inhibitors. BMC Cancer. 2008; 8: 237-242. [CrossRef]

[4] Kim MS, Kim JE, Lim DY, Huang Z, Chen H, Langfald A, Lubet RA, Grubbs CJ, Dong Z, Bode AM. Naproxen induces cell-cycle arrest and apoptosis in human urinary bladder cancer cell lines and chemically induced cancers by targeting PI3K. Cancer Prev Res. 2014; 7(2): 237-245. [CrossRef]

[5] Lubet RA, Scheiman JM, Bode A, White J, Minasian L, Juliana MM, Boring DL, Steele VE, Grubbs CJ. Prevention of chemically induced urinary bladder cancers by naproxen: protocols to reduce gastric toxicity in humans do not alter preventive efficacy. Cancer Prev Res. 2015; 8(4): 297-302. [CrossRef]

[6] Kömürcü ŞG, Rollas S, Ulgen M, Gorrod JW, Cevikbaş A. Evaluation of some arylhydrazones of p-aminobenzoic acid hydrazide as antimicrobial agents and their in-vitro hepatic microsomal metabolism. Boll Chim Farmaceutico. 1995; 134: 375-379.

[7] Küçükgüzel ŞG, Oruc EE, Rollas S, Şahin F, Ozbek A. Synthesis, characterization and biological activity of novel 4thiazolidinones, 1,3,4-oxadiazoles and some related compounds. Eur J Med Chem. 2002; 37: 197-206. [CrossRef] 
[8] Küçükgüzel ŞG, Mazı A, Şahin F, Ozturk S, Stables J. Synthesis and biological activities of diflunisal hydrazidehydrazones. Eur J Med Chem. 2003; 38: 1005-1013. [CrossRef]

[9] Rollas S, Küçükgüzel ŞG. Biological activities of hydrazone derivatives. Molecules. 2007; 12: 1910-1939. [CrossRef]

[10] Çıkla P, Özsavcı D, Bingöl Özakpınar Ö, Şener A, Çevik O, Özbaş Turan S, Akbuğa J, Şahin F, Küçükgüzel ŞG. Synthesis, cytotoxicity and pro-apoptosis of etodolac hydrazide derivatives as anticancer agents. Arch Pharm. 2013; 346: 367-379. [CrossRef]

[11] Çıkla P, Tatar E, Küçükgüzel İ, Şahin F, Yurdakul D, Basu A, Krishnan R, Nichols DB, Basu NK, Küçükgüzel ŞG. Synthesis and characterization of flurbiprofen hydrazide derivatives as potential anti-HCV, anticancer, antimicrobial agents. Med Chem Res. 2013; 22: 5685-5699. [CrossRef]

[12] Aydın S, Basu NK, Arora P, Basu A, Nichols DB, Talele TT, Akkurt M, Celik İ, Buyukgungor O, Küçükgüzel ŞG. Microwave assisted synthesis of some novel flurbiprofen hydrazide-hydrazones as anti-HCV NS5B and anticancer agents. Marmara Pharm J. 2013; 17: 26-34. [CrossRef]

[13] Küçükgüzel ŞG, Koc D, Cıkla P, Özsavcı D, Ozakpınar OB, Tiber PM, Orun O, Erzincan P, Erdem SS, Şahin F. Synthesis of tolmetin hydrazide-hydrazones and discovery of a potent apoptosis inducer in colon cancer cells. Arch Pharm. 2015; 348: 730-742. [CrossRef]

[14] Şenkardeş S, Basu NK, Durmaz İ, Manvar D, Basu A, Atalay R, Küçükgüzel ŞG. Synthesis of novel diflunisal hydrazide-hydrazones as anti-hepatitis $\mathrm{C}$ virus agents and hepatocellular carcinoma inhibitors. Eur J Med Chem. 2016; 108: 301-308. [CrossRef]

[15] Sarıözkan S, Gaffari T, Pelin CS, Güvenç M, Yüce A, Yay A, Cantürk F, Küçükgüzel ŞG. Effect of etodolac hydrazone, a new compound synthesized from etodolac, on sperm quality, testicular lipid peroxidation, apoptosis and sperm DNA integrity. Andrologia. 2016; 48: 177-88. [CrossRef]

[16] Tatar E, Şenkardeş S, Sellitepe HE, Küçükgüzel ŞG, Karaoğlu ŞA, Bozdeveci A, De Clercq E, Pannecouque C, Hadda TB, Kucukguzel İ. Synthesis, and prediction of molecular properties and antimicrobial activity of some acylhydrazones derived from N-(arylsulfonyl)methionine. Turk J Chem. 2016; 40: 510-34. [CrossRef]

[17] Tok F, Beyhan N, Erzurumlu Y, İlhan R, Ballar P, Kaymakcığlu BK. Antiproliferative activity of some tautomeric hydrazones derived from chalcones. Marmara Pharm J. 2016; 20: 157-163. [CrossRef]

[18] Keogh A, Şenkardeş S, Idle JR, Küçükgüzel ŞG, Beyoğlu D. A novel anti-hepatitis C virus and antiproliferative agent alters metabolic networks in HepG2 and Hep3B Cells. Metabolites. 2017; 7: 2-16. [CrossRef]

[19] Popiołek L, Biernasiuk A. Synthesis and investigation of antimicrobial activities of nitrofurazone analogues containing hydrazide-hydrazone moiety. Saudi Pharm J. 2017; 25: 1097-1102. [CrossRef]

[20] Küçükgüzel ŞG, Küçükgüzel İ, Tatar E, Rollas S, Şahin F, Güllüce M, De Clercq E, Kabasakal L. Synthesis of some novel heterocyclic compounds derived from diflunisal hydrazide as potential anti-infective and anti-inflammatory agent. Eur J Med Chem. 2007; 42: 893-901. [CrossRef]

[21] Mentese MY, Bayrak H, Uygun Y, Mermer A, Ülker S, Karaoğlu SA, Demirbaş N. Microwave assisted synthesis of some hybrid molecules derived from norfloxacin and investigation of their biological activities. Eur J Med Chem. 2013; 67: 230-242. [CrossRef]

[22] Kaplancıklı ZA, Yurttaş L, Özdemir A, Turan-Zitouni G, Çiftci GA, Yıldırım SU, Mohsen UA. Synthesis and antiproliferative activity of new 1,5-disubstituted tetrazoles bearing hydrazone moiety. Med Chem Res. 2014; 23: 1067-1075. [CrossRef]

[23] Küçükgüzel ŞG, Çıkla Süzgün P. Recent advances bioactive 1,2,4-triazole-3-thiones. Eur J Med Chem. 2015; 97: 830870. [CrossRef]

[24] Çıkla Süzgün P, Basu NK, Basu A, Arora P, Talele TT, Durmaz İ, Çetin Atalay R, Küçükgüzel ŞG. Anti-cancer and anti-hepatitis C virus NS5B polymerase activity of etodolac 1,2,4-triazoles. J Enzyme Inhib Med Chem. 2015; 30(5): 778-785. [CrossRef]

[25] Tatar E, Küçükgüzel ŞG, Karakuş S, De Clercq E, Andrei G, Snoeck R, Pannecouque C, Okullu SÖ, Ünübol N, Kocagöz T, Kalaycı, Şahin F, Küçükgüzel İ. Synthesis and biological evaluation of some new 1,3,4-thiadiazole and 1,2,4-triazole derivatives from L-methionine as antituberculosis and antiviral agents. Marmara Pharm J. 2015; 19: 88-102. [CrossRef]

[26] Kulabaş N, Tatar E, Özakpınar ÖB, Özsavcı D, Pannecouque C, De Clerck E, Küçükgüzel İ. Synthesis and antiproliferative evaluation of novel 2-(4H-1,2,4- triazole-3-ylthio)acetamide derivatives as inducers of apoptosis in cancer cells. Eur J Med Chem. 2016; 121: 58-70. [CrossRef] 
[27] Harrington PJ, Lodewijk E. Twenty Years of Naproxen Technology. Organic Process Research \& Development 1997; 1: 72-76

[28] European Pharmacopoeia 7.0, Monographs N-O, p.2554-2555.

[29] Cumaoğlu A, Dayan S, Agkaya AO, Özkul Z, Özpozan NK, Synthesis and pro-apoptotic effects of new sulfonamide derivatives via activating p38/ERK phosphorylation in cancer cells. J Enzyme Inhib Med Chem. 2015; 30(3): 413-419. [CrossRef]

[30] http://www.molinspiration.com (accessed March 14, 2018)

[31] Zhao Y, Abraham MH, Lee J, Hersey A, Luscombe NC, Beck G, Sherborne B, Cooper I. Rate-limited steps of human oral absorption and QSAR studies. Pharm Res. 2002; 19: 1446-1457.

[32] Harrison IT, Lewis B, Nelson P, Rooks W, Roszkowski A, Tomolonis A, Fried JH. Nonsteroidal antiinflammatory agents. I. 6- Substituted 2-naphthylacetic acids. J Med Chem. 1970; 13 (2): 203-205.

[33] Amir M, Kumar H, Javed SA. Non-carboxylic analogues of naproxen: design, synthesis, and pharmacological evaluation of some 1,3,4-oxadiazole/ thiadiazole and 1,2,4-triazole derivatives. Arch Pharm Chem Life Sci. 2007; 340: 577-585. [CrossRef]

This is an open access article which is publicly available on our journal's website under Institutional Repository at http://dspace.marmara.edu.tr. 